\title{
QUÉ PIENSAN LOS PROFESORES ACERCA DE CÓMO SE DEBE ENSEÑAR
}

\author{
FERNÁNDEZ GONZÁLEZ, J. ${ }^{1}$ y ELORTEGUI ESCARTÍN, N. ${ }^{2}$ \\ 1 IB Cabrera Pinto, La Laguna, Escuela Universitaria de Formación del Profesorado. \\ Universidad de La Laguna. \\ ${ }^{2}$ IPFP de Santa Cruz de Tenerife.nelorte@ibm.net
}

\section{SUMMARY}

Authors propose different types of teacher after working with a group of Primary and Secondary teachers in the Science area. In a structuralistic way, differentiating elements were determinated and «behaviour models in teaching/learning tasks" were established with the intention of expliciting theories behind each practice and, therefore, opening the way to its analysis and evolution.

\section{INTRODUCCIÓN}

Las concepciones de los profesores evolucionan siguiendo un complejo camino de desarrollo que podríamos equiparar a la evolución de las especies de Darwin, pero aquí referida a la evolución del pensamiento de los profesores sobre lo que se debe y se puede enseñar. Esta concepción evolutiva ha quedado de manifiesto en los trabajos de distintos escuelas del pensamiento didáctico, en algunos casos de forma explícita (Tuolmin, 1972) y, en otros, de forma implícita.

Hay un acuerdo generalizado en aceptar la existencia de diversos caracteres que definen a distintos educadores, en delimitar determinados tipos de profesores y en perfilar los avances y retrocesos de algunas formas de ejercer la docencia (Brincones, 1986; Rozada, 1985; Host, 1988; Escudero, 1981) o en criticar determinadas concepciones relativas al ejercicio de la docencia (Gil, 1983,1993 ).

Podemos encontrar signos de esta evolución si examinamos lo sucedido en el sistema educativo español en los últimos quince años. Han ido emergiendo diferentes tipos de profesor para adaptarse a las variaciones del entorno educativo. Estas variaciones están asociadas a la asimilación de ciertas ideas referidas a la educación por parte del estamento docente. Una vez aceptada una nueva idea, el profesorado se adapta a la nueva situación, incorpora la nueva concepción a su práctica profesional $y$ evoluciona.

La aplicación de la Ley General de Educación de 1970 afectó casi exclusivamente el volumen y estructura del sistema educativo, a pesar de las voces que intentaron aprovechar la situación para impulsar un cambio en la metodología del profesorado. Al no prosperar estas propuestas, permaneció casi indiscutible la figura del docente transmisor del conocimiento y de comunicación unidireccional hasta que se producen dos hechos en ta primera mitad de los ochenta que alumbran nuevos tipos de profesor: la consolidación de los Movimientos de Renovación Pedagógica (MRP) y la experimentación de la primera reforma.

Como consecuencia de estos hechos, a principios de los ochenta encontramos, al menos, cuatro tipos diferentes de profesor, a los cuales vamos a describir de forma somera, para profundizar en su análisis a lo largo de este trabajo: 
- El tipo de profesor que hemos citado anteriormente y que, según diferentes autores se suele denominar tradicional, «de siempre», transmisor o transmisor-receptor. Nosotros, en lo sucesivo, hablaremos de profesor «transmisors.

- El tipo de profesor autodidacta en cuestiones pedagógicas, que elabora su forma de trabajo a partír de su propia experiencia profesional en el aula. Este docente, en una búsqueda profesional autónoma, acumula un amplio repertorio de recursos profesionales, desarrolla su librillo sin apenas tener en cuenta influencias externas procedentes de otros campos del conocimiento, a veces compartiendo experiencias con compañeros. En diferentes fuentes se asocia a este modelo las denominaciones de artesano, humanista, activista, practicista, artista, naturalista y de estructuración-construcción. Para este profesor usaremos la denominación de «artesano».

- El profesor que proviene de la explosión de la pedagogía por objetivos y que fomenta el trabajo docente con un fuerte componente tecnológico. Para este tipo de profesor, la base de una enseñanza eficaz está en la planificación y el control de cada variable que pueda afectar el trabajo en el aula. En la bibliografía se encuentra como profesor técnico, tecnocrático, tecnológico, eficaz, tecnicista y transmisor-estructurado y; en lo sucesivo, a este tipo de profesor lo llamaremos «tecnológico».

- El tipo de profesor que sigue la corriente del pensamiento empirista y se basa en la idea de que el alumno es capaz de reelaborar el conocimiento de cada disciplina, si se le pone en situación de recrear los momentos fundamentales de cada ciencia, y que cree que el desarrollo de tos procesos cognitivos principales -hipótesis, deducciones, planificaciones, etc.- debe ser autónomo. En trabajos de otros autores denominan a este modelo descubridor, de descubrimiento o investigativo. Nosotros usaremos la denominación de «descubridor».

Fistos cuatro tipos de profesor conviven en mayor o menor grado en la innovación, en la renovación del sistema educativo español, en los congresos y en la investigación educativa, pero bastante menos en la realidad del aula donde, en mayor medida, el profesor «transmisor» conserva su estatus predominante.

Fin tiempos más recientes aparece un nuevo tipo de profesor, con una base fuertemente psicológica, para el que la determinación de lo que sucede en la cabeza del alumno y el trabajo sobre sus esquemas mentales constituyen la base de un buen aprendizaje y que suele ser identificado como constructivista, de elaboración, crítico, elaborador, reflexivo e investigador en el aula. Vamos a utilizar la denominación de «constructor».

Los autores de este trabajo, al analizar este proceso, observan un mundo educativo que está a medio camino en la elaboración de un cuerpo de conocimiento que fundamente la tarea docente. Consideramos que, ante tantas variantes, es necesario conceptualizar y caracterizar cada una de ellas y explorar el pensamiento docente que las sustenta; de esta manera, se dispondría de un instrumento de análisis de la práctica docente y de una fundamentación para el desarrollo profesional.

\section{DISTINTAS FORMAS DE ENSEÑAR: MODELO DIDACTICO, TEORIA Y PRÁCTICA, PENSAMIENTO Y ACCIÓN: ¿POR QUE ACTUAMOS?}

E] trabajo como docente tiene un carácter eminentemente práctico. Pero no debe ser sólo eso. Detrás de la práctica debe haber un cuerpo teórico que explique en qué fundamento se basa.

Destaquemos la idea de que cualquier práctica gue un individuo realiza en su vida responde siempre a una teoría. Toda práctica como ciudadano, como padre, como profesor, etc. responde siempre a una teoría implícita. No existe la posibilidad de realizar ningún tipo de acción sin que tenga su correlación teórica cognitiva que la justifique. Ahora bien, aunque toda práctica conlleva una teoría, no significa que siempre que hacemos algo lo hagamos conscientes de cuál es el encuadre teórico que lo respalda (Porlán, 1983).

EI avance que supone la incorporación de la teoría al trabajo práctico y cotidiano deja bien confirmado aquello de que no hay nada más práctico que una buena teoria.

Cuando se tiene una profesión como la docente, en la que hay que intervenir socialmente por cuanto se interacciona con otras personas (alumnos, compañeros, padres, etc.), se está inevitablemente marcado por las concepciones ideológicas personales para juzgar todo el proceso de enseñanza-aprendizaje que se realiza, aunque sea una ideología elemental y de práctica inconsciente.

Así, toda modelización en el campo de la enseñanza tiene un componente teórico y un componente ideologico de los que se debe ser consciente, lo que conlleva inevitablemente la pluralidad de modelos.

La palabra «modelo» en didáctica aparece como muestra o estereotipo de posibie alternativa a la enseñanzaaprendizaje. Como cualquier otro, los modelos didácticos son una interpretación de la realidad que sólo tienen validez en un campo de aplicación determinado, pero cuya interpretación suele ser inexacta fuera de los límites de utilidad; es decir, tienen un rango de validez.

El modelo es un esquema mediador entre la realidad y el pensamiento, una estructura en torno a la que se organiza el conocimiento y tendrá siempre un carácter provisional y aproximativo a la realidad (Gimeno, 1981). La comprensión de los casos reales se ha de hacer mediante el solapamiento de las idcas de dos o más modelos teóricos. El modelo didáctico es un recurso para el desarrollo técnico y la fundamentación científica de la enseñanza, que intenta evitar que continúe siendo «una 
forma empirica y particular» alejada de cualquier formalización (Martínez Santos, 1989).

Para Cañal-Porlán (1987), los modelos didácticos son la «construcción teórico formal que, basada en supuestos científicos, idecológicos y sociales, pretende interpretar la realidad y dirigirla hacia unos determinados fines educativos».

Fistas reflexiones nos muestran que no disponemos de una buena teoría, ya que estamos a medio camino en la construcción de un cuerpo de conocimiento que fundamente los procesos de enseñanza y de aprendizaje. Habrá que conformarse, de momento, con caracterizar los modelos más directamente observables.

\section{NUESTRO TRABAJO}

Pretendemos presentar algunas de las formas de caracterizar «cómo enseñar ciencias»; es decir, las pautas para la enseñanza de contenidos científicos y las implicaciones de contorno de estas condiciones expresadas en código de lenguaje didáctico. No creemos posible ofrecer mecanismos ni soluciones lineales ni fáciles, ya que la gran complefidad de la enseñanza de las ciencias nos permite únicamente teorizar acerca de las situaciones más frecuentes, con la intención de elaborar, para los profesores, herramientas (en forma de categorías) que les permitan afrontar con mayor conocimiento la situación en el aula.

La obtención de datos para la elaboración de los modelos se realizó, durante varios años, sobre un colectivo de profesores de EGB y secundaria (BUP y FP), asistentes a actividades de formación y perfeccionamiento, que exigían su posicionamiento didáctico y metodológico. Este tipo de actividades nos permitió recoger y analizar un gran volumen de datos acerca de cómo enseñan los profesores, poco sistemáticos inicialmente, pero que nos sirvieron para empezar a separar diferentes pautas de comportamiento en el aula.

En analogía con otros procesos de la reflexión teórica sobre la práctica, tratamos los datos recogidos desde una concepción de la modelización de los comportamientos profesionales.

Para hacer la modelización, en primer Iugar se seleccionaron elementos diferenciadores de distintos profesores que pudieran actuar como indicadores de diferentes concepciones y prácticas docentes. A continuación, sobre el estudio del primer conjunto de datos procedentes de entrevistas o charlas abiertas se fueron perfilando, hasta conseguir completarlos, unos cuadros emergentes dc enseñanza-aprendizaje. En este proceso se fueron reformulando algunas de nuestras observaciones iniciales y pudimos hacer una primera agrupación de profesores representativos de cinco formas diferentes de entender cl quehacer del aula. La agrupación en cinco modelos nos permitió encajar, de forma coherente, tanto los datos obtenidos en el trabajo con los profesores, como las descripciones procedentes de la bibliografía disponible.

Usando como indicadores los elementos diferenciadores de cada modelo, se planificó una segunda, y hasta una tercera entrevista abierta, dirigidas a personas específi. cas que representaban a un grupo. Con estas nuevas entrevistas se consiguió completar gradualmente la cáracterización de los grupos que habíamos asociado a los iipos de profesor.

Finalmente, de cada uno de los cinco grupos, seleccionamos a un miembro como elemento característico con el que poder perfilar «sus formas de enseñar» y su manera de interpretar los complejos procesos en el aula.

Para aclarar las características de cada modelo, además de nuestras observaciones, incluimos algunos de los comentarios más significativos que hicieron los profesores durante las entrevistas.

\section{CÓMO HEMOS CARACTERIZADO UN MODELO?}

La caracterización de un modelo didáctico supone la selección y estudio de los principales aspectos asociados al pensamiento del docente y a la práctica educativa. Estos aspectos son muy variados y numerosos. Por ello se han separado según afecten más directamente a la teoría o a la práctica docente, relacionando cada grupo de concepciones con el de problemas y cuestiones que lleva asociado. Todo esto estructura las entrevistas en las que se basa nuestro trabajo. (Ver el cuadro de la página siguiente).

En la práctica, no encontramos versiones puras de un modelo, sino que detectamos entremezclados ciertos rasgos característicos de varias tipologías de docente en un mismo individuo o grupo. No obstante, Ios modelos nos permitirán analizar el trasfondo que sustenta sti actividad profesional.

Dada la extensión de las caracterizaciones que acompañan a cada modelo didáctico, reflejamos en este artículo únicamente las opiniones más dircctamente relacionadas con la metodología utilizada por cada modelo (cómo enseñar), omitiendo los demás aspectos fundamentales señalados.

\section{CÓMO SE ENSEÑA EN LOS DISTINTOS MODELOS DIDACTICOS}

\section{El profesor «transmisor»}

El modelo de profesor «transmisor» enseña siguiendo un esquema muy parecido al que ha estado sometido duran+ te todo su período de formación inicial, incluso desde su época de adolescente. 


\section{CONCEPCIONES}

\section{Teoría docente}

- Concepciones sobre el conucimiento científico, la filosotía y la historia de la ciencia o sobre la estructura de las disciplinas cientificas.

Concepciones sociológicas: ideología política y social, la estructura de poder en el aula, la visión de la educación o la visión de la profesión docente.

- Concepciones psicológicas: concepción de la psicología de los alumnos, papel de la motivación, posicionamiento ante las diferentes teorias de la psicología del aprendizaje.

\section{Práctica dacente}

-. Concepciones metodológicas: uso de recursos, documentación, mecanismos de comunicación con los alumnos, organización del trabajo en el aula y en su entorno, tipo y estructura de las actividades y problemas con que se trabaja.

- Concepciones sobre la planificación de la enseñanza: objetivos, conteniłos, procedimientos, secuenciación, cutrículo.

-.. Concepciones sobre la formación y perfeccionamiento: asesoramiento, investigación educativa, formación inicial.

- Concepciones sobre la evaluación.

\section{PROBLEMAS Y CUESTIONES ASOCIADAS}

- ¿Qué es la ciencia? ¿Cómo se desarrollo y su đesarrolla la ciuncia? ¿Qué características tiene el trabajo cientifico? ¿En qué consiste el método científico? ¿ Como se relaciona el conocimiento cotidiano, el cientifico y el escolar?

- Cuâl es el papel de la escuela en la sociedad? ; Y el de la ideología en la escuela? ¿Para qué la escuela? ¿Cuáles son las metas o fines do lo que hacemos? ¿Por qué hacemos to que hacemos? ¿Qué enseñar? ¿Cómo es quien enseña? ¿Cómo debe ser quien enseña?

¿Como aprenden los alumnos? ¿Cómo son los alumnos? ¿Cuáles son sus intereses y motivación y hasta donde debemos atenderlos? ¿Cómo estimular el interés de los alumnos?

- ¿Qué tipo de documentos prevalecen en la enseñanza? ¿Cúmoes el uso del libro de texto, apuntes y bibliografía? ${ }_{\text {¿Como intervienen los }}$ medios audiovisuales?

- ¿Como conjugar todo en el aula? ¿Cómo transcurre un día de clase'? ¿Como es la comunicación en el aula? ¿Cómo se organiza a los alumnos?

- ¿Qué actividades hacernos? ¿Que problemas plantearemos? ¿Actividades/problemas para qué? ¿Cómo son las actividades que se realizan? ¿Qué papel juega el trabajo de laboratorio? ¿Como se hace el rabajo de laboratorio?

- ¿Qué papel juegan los objetivos en la enseñanza? ¿Cómo se conjuga la idea de objetivo y de contenido? Cómo se relacionan los procedimientos con los objetivos y contenidos?

- ¿Cuál cree que debe ser el papel del asesor docente? ¿Cómo entiende que se perfecciona?

- ¿Cómo entiende la investigación en la educación? ¿Quién la debe hacer: la universidad, los profesores de aula en la universidad, los propios profesores en el aula?

$-¿$ Como debe formarse al futuro profesor?

¿Qué entiende por "el nivel de los alumnos»? ¿Qué entiende por "evaluación objetivas? ¿La evaluación ucasiona un nuevo planteamiento del trabajo del profesor? ¿Qué se evalúa? 'Cómo evaluar? ¿Para qué evaluar? ¿A quién? ¿Cuándo? ¿Con qué? ¿Qué papel tienen las pruebas, exámenes, controles y tosts? ¿Como se hace la recuperacion?
Para este tipo de profesor, la asignatura está ya organizada y su prioridad es el cumplimiento de la programacíon oficial, dejando para «Ios expertos» la tarea de decidir la validez o no de cómo enseñar la asignatura.

«El orden en los conceptos de la disciplina es la manera normal de hacer las cosas.»

«La secuencia y cl contenido de la asignatura lo definen la propia estructura de la física y la química. Además, las programaciones oficiales y el examen de selectividad ya se han ocupado de eso y, al fin y al cabo, las preparan expertos. Yo sé el tiempo que tengo y sé cuántos temas tengo que enseñar, ásí que me limito a repartir el tiempo disponible. Claro que eso hace que no me pueda permitir enrollarme en disquisiciones con los alumnos si hay preguntas complicadas, después de clase. Si no, no hay quien acabe el programa.»

Los alumnos trabajan individualmente, en un sólo grupo y con un fuerte componente «igualitarion: el trato y Ia exigencia debe ser idéntica para todos. El protesor se relaciona con los alumnos a través de una comunicación 
unidireccional desde «el que sabe hacia el que es lego», mediante un exposición mayoritariamente verbal con soporte escrito. Considera que poca o ninguna información relevante puede recibirse de ellos más allá de un no entiendo. La clase es únicamente responsabilidad del profesor que con su explicación liena toda la clase y ocupa todo su tiempo. El orden de la clase es fundamental para evitar las distracciones, alborotos, conversaciones y llamadas de atención.

Es el prototipo dominante dentro y fuera del sistema educativo, y todos los profesionales de la enseñanza estamos, inevitablemente, influidos en mayor o menor manera por él: la transmisión verbal de conocimientos es la forma habitual de enseñar en los centros educativos (Porlán, 1993).

¿Cuantas manías, gustos y estrecheces hemos tenido que averiguar los alumnos en algunos profesores para poder superar la asignatura!

«Los alumnos deben trabajar individualmente en clase. Cuando hablan entre ellos se distraen y además no me atienden.»

«Pretender que trabajen juntos solo lleva a que los más listos sc retrasen y los menos listos no avancen. Además, en la vida diaria hay que enfrentarse solo a examenes, oposiciones y todo tipo de prueba, así que más vale que se vayan acostumbrando.»

«La obligación de los alumnos en clase es la de atender y tomar apuntes. Al fin y al cabo, yo soy el que explica y ellos pueden hablar cuando les pregunto si han comprendido. Pero son muy poco participativos.»

«Es una metodología activa porque el alumno tiene que estar continuamente atento y siguiendo activamente las explicaciones en la pizarra.»

«Para enseñar es suficiente con el dominio de la disciplina; el que sabe, sabe enseñar.»

La documentación que utiliza es, casi en exclusiva, el libro de texto y «unos buenos apuntes» dictados o guiados por el profesor, que se consideran de una autoridad incuestionable $y$, por tanto, el trabajo fundamental deI alumno es que aquéllos sean lo más fidedignos posibles y sean incluso implementados con otras informaciones (libros, enciclopedias, etc.). Los apuntes que se dan deben estar acordes con el nivel de exigencia del curso que se imparte, bien explicados siguiendo la lógica disciplinar. Si los alumnos siguen las explicaciones atentos y copian bien los apuntes, aprueban todos.

En cuanto a otros recursos materiales, utiliza principalmente la pizarra, aunque ha ido aceptando el uso del vídeo en lugar de la explicación como una nueva forma de exposición. El grupo se organiza en una pequeña aula donde quepan unos pupitres estáticos «para mayor orden.»

«Los alumnos se basan en los apuntes que toman y en el libro de texto. Ellos no saben redactar suficientemente y, además, suclen anotar muchos disparates. Al fin y al cabo, el libro es lo que queda y siempre podrán acudir a él mañana."

«Los alumnos fracasan porque no saben tomar apuntes. Los alumnos buenos tienen buenos apuntes»
«Desde luego, yo solo no puedo elaborar un material como el que prepara una editorial y sus autores. Strele haber cosas que no me gustan, pero entonces yo las explico a mi manera en lugar de seguir el libro.»

«Generatmente explico en la pizarra, aunque algunas cosas se ven bien en algunos vídeos que hemos tóo juntando en el departamento. Si están bien hechos, algunos vídeos explican muy bien las cosas, y siempre puede uno complementarlos con la propia explicación,»

Respecto a las actividades que pone en práctica, trabaja ampliamente en la resolución de ejercicios con un enunciado cerrado, con hincapié mecantcista en los algoritmos y aplicación de modelos matemáticos; el dominio se consigue a través de la resolución de ciertos «tipos» que aparecen en hojas de problemas, por temáticas, para que los resuelvan los alumnos.

En general se carece de parte experimental y, en caso de existir, predomina la demostración magistral con una gran carga de aprendizaje técnico o la comprobación de lo visto, previamente, en una explicación de teoría. Hay una fuerte separación de teoría y práctica.

"A parte de lasexplicaciones de la teoría, solemos hacer problemas y algunas experiencias de laboratorio.»

«Para los problemas, normalmente los del Jibro estân bien, aunque también tengo hojas de problemas que les doy fotocopiadas y que saco de otros libros.»

"Tenemos una serie de problemas-tipo que deben saber solucionar, porque son la base de los siguientes cursos."

«Hay algunas prácticas básicas que las hacen los alumnos en el laboratorio con los equipos del Ministerio. Pero no suelen salir porque los chicos no entienden los guiones de los equipos.»

«La verdad es que se saca poco rendimiento de las practicas, hay que tener mucha habitidad para que salgan, y para ellos es frustrante que salgan mal. Es preferible hacerles una demostracion magistral bien preparada, que incluso permite obtener datos que confirman la teoria.»

El planteamiento de dificultades como situaciones de difícil solución a través de problemas suele evitarse porque «no tienen solución.»

\section{EI profesor «tecnológico»}

El modelo de profesor "tecnológico», parece ser al que tiende mucho profesorado que se siente insatisfecho con el modelo anterior y quiere mejorar el cómo enseñar.

Para este estereotipo, todo el quehacer de la enseñanza debe estar mediatizado por el método científico (observación, hipótesis, experimentación y teoría) y, por tanto la organización didáctica debe estar "normalizada»; es decir, en ella debe estar detallado «qué es lo que se đebe hacer.»

La planificación de la enseñanza suele estar basada en una programación cerrada, con fuerte arraigo en la secuenciación de objetivos (generales, específicos, temá- 
ticos, operativos, terminales, etc.) dirigidos a adquirir conocimientos y capacidades según la lógica de la disciplina. Es necesario poner objetivos, «lo que se debe aprender", para conocer las intenciones de lo que enseกีamos.

¿Cuánto mejor hecha esté la programación y cuánto más se cumpla, siguiendo todas las actividades en su totalidad, más cerca estará el éxito de la enseñanza!

El profesor utiliza muchas «fichas» de propuesta de trabajo para el alumno, lo que conlleva actividades muy cerradas y con una secuencia de dependencia las unas de las otras. Hay un proceso gradual en la enseñanza de los contenidos de la ciencia y la secuencia de los objetivos es una forma de escalonar los conceptos en niveles de complejicad para facilitar el aprendizaje.

«Una enseñanza eficaz debe tener en cuenta todas las variables que inciden en el aula. De esa manera, todo estará controlado y el rendimiento es el mejor.»

«Hay que definir cuidadosamente qué se pretende: cuáles son los objetivos de cada actividad y cuáles son los objetivos terminales de cada curso. Tener bien definidos los objetivos te permite no desviarte en clase y cumplir lo programado.»

«Es que los programas del Ministerio son muy extensos. Si lo hago de otra manera, no me da tiempo a dar el programa."

"Tienes que secuenciar los objetivos de manera que cada paso te permita poner las bases del siguiente. $Y$ para eso el mejor orden de los contenidos es el de siempre: cinemática, dinámica, energética, electricidad, etc.»

«Siempre hay alumnos que intentan que pierdas el tiempo con preguntas tontas para que no avances en el programa, pero si estás prevenido y tienes bien planeado lo que quieres, nomalmente todo funciona.»

Este modelo se relaciona con los alumnos de diversas formas (verbal, audiovisual, prensa escrita, medios de comunicación, etc.), pero se trata de comunicación dirigida por el profesor. Predomina la lección magistral como forma idónea de enseñanza, aunque esta metodología expositiva suele estar acompañada de una participación socrática que avala el quehacer del profesor. Sólo existe un grupo de estudiantes, «todos iguales», con los que el profesor intercambia su actividad.

«Trabajan individualmente, porque los objetivos minimos que tengo definidos los tiene que superar cada uno.»

«En el laboratorio trabajan en grupos porque no tengo equipos suficientes, pero deben obtener los datos experimentales individualmente siempre que se pueda, para yo poder ver qué es lo que cada uno supera.»

«Básicamente yo explico, pero utiłizamos vídeos o noticias del periodico que selecciono y preparo, y entonces hay algunos debates en que actúo socráticamente, haciéndoles preguntas. Lo malo de esto es que tienes muy poco control sobre cómo se desarrolla en ta clase, y en cuanto te desvías tienes que terminar cortando y volviendo a la explicación.»

El material acorde con este modelo consta de fichas o guías muy programadas para profesores y para alumnos, bajo epígrafes como "guía del profesor», "guía del alumno», tema tal o cual. Aparece todo calculado, pro- gramado y previsto, tanto de textos como de apuntes. In cuanto a otros recursos materiales, la pizarra y el vídeo son recursos habituales, aunque se requiere estar dotado de material específico y avanzado, tanto para la experimentación como para otras propuestas: ordenador, $\mathrm{pH}$ metro, cromatógrafos, etc.

"Además del libro de texto preparo guiones que describan el procedimiento de to que tienen que hacer, especialmente si vamos a hacer alguna práctica. Hay que detallar mucho, porque se pierden."

"Cuando encuentro alguna noticia del periódico que merece la pena, la preparo con un cuestionario y se hace en clase, pero les cuesta muchísimo sacar conclusiones de las noticias del periódico, leen muy mal."

«Además de la pizarra, se trabaja sobre los guiones o las fichas que tengo preparadas y que incluyen las cuestiones qque orienten la actividad hacia el objetivo de ésta.»

«He visto algunos programas de ordenador que irían estupendamente porque están muy bien estructurados y guian perfectamente al alumno, lo que pasa es que no tenemos ordenadores suficientes. Pero el futuro está en la enseñanza asistida por ordenador; por ahí fuera ya está establecido.»

Se acometen con gran intensidad las prácticas de laboratorio, que están orientadas por un material específico. Son prácticas comprobatorias de algunas situaciones de lo que se enseña en teoría. Se va del aula al laboratorio. Estas prácticas están estructuradas en guiones descriptivos del procedimiento, empleando recetas pormenorizadas.

Otra de las facetas muy cultivadas es la resolución de ejercicios, entendidos como trabajos de cálculo con solución cerrada. A veces se introducen algunos ejerctcios problemáticos de resultado desconocido, pero que tienen solución bajo condiciones supuestas.

«Resolvemos problemas en clase como aplicación de la teoría Los saco de libros, aunque a veces me los invento. Lo difícil es redactar bien el entunciado, y además es conveniente elegir bien los datos iniciales para que los resultados queden bien, con números redondos, que siempre son más fáciles de manejar.»

«Hacemos prácticas de laboratorio que tenemos muy bien preparadas; cada una con su receta muy detallada, y que salen bien."

«En la medida de fo posible, los principales conceptos de la teoría procuramos comprobarlos con alguna práctica, aunque no es fácil encontrar prácticas en las que salgan datos suficientemente buenos.»

\section{El profesor «artesano»}

Este estereotipo, lo adoptan algunos docentes insatisfechos con el modelo tradicional, decepcionados de las estructuras educativas y de las posiciones académicas dogmáticas. Es frecuente en las etapas iniciales de! sistema educativo.

Se caracteriza por la ausencia de cualquier planificación. Se hace gran hincapié en la actividad autónoma de 
los alumnos y se desecha cualquier dirección de aprendizaje.

En estecaso, la organización de los procesos de enseñanza-aprendizaje se basa en sus rutinas; no se explicitan las intenciones reales e incluso se aparenta que no existen finalidades didácticas en la planificación. El quehacer se deja gobernar por los métodos de trabajo del docente, que son espontáneos, sin secuencia, sin seguir una concepción cientificista de observación, de hipótesis, de experimentación y conclusiones. Es disciplinar y tiende a lia interdisciplinariedad.

«Creo que el valor de la programación es muy relativo; uno debe tener claro de qué ya a hablar pero entrar en detalles es una pérdida de tiempo, porque lo que va surgiendo en clase cambia totalmente lo que hubieras pensado previamente.»

«Sin la motivación de los alumnos, éstos no pueden tener un aprendizaje significativo, y esà motivación está claramente definida por sus intereses. Por tanto, son sus intereses los que definen el trabajo en clase.»

"La verdadera utilidad de mi programación es la de saber claramente la secuencia de actividades que utilizo. Esa secuencia la tengo elaborada en función de lo que pretendo, de a donde quiero llegar.»

«El mundo real no tiene el conocimiento separado cn disciplinas estancas entre sí. Con los alumnos se debe trabajar en temas que tiencn alrededor y según su propio punto de vistá y su interés: su barrio, la naturaleza, la contaminación, los alimentos y las dietas, etc.,

La comunicación con sus alumnos es muy abierta, predominantemente interactiva y espontánea, lo que favorece la riqueza participativa del alumno y la manifestación de su personalidad de forma natural.

La organización de la clase no sigue ningún esquema prefijado. Se improvisan las situaciones, se alternan el grupo-clase completo y el grupo reducido de alumnos, mientras los demás chicos actúan por libre iniciativa. La práctica de organizar grupos regulares no es nunca un requisito, salvo que los propios alumnos los induzcan por sus amistades, aficiones, etc.

«Ellos se organizan a su manera. Si alguien propone trabajar en un tema que interesa a varios, ésos forman un grupo para este estudio y, cuando cambian de tema, cambian la organización. Al fin y al cabo, a mí no me afecta cómo se agrupan.»

«Solemos trabajar en cosas que ellos proponen y con sus propios diseños. A veces los diseños no llevan a ninguna parte y yo lo veo desde el principio, pero de sus propios errores aprenden tanto como de los aciertos."

«En clase dejo mucha libertad para intervenir. Al principio les cuesta, pero en unos meses se sienten cómodos interviniendo y hay largas secuencias de preguntas, respuestas y nuevas preguntas. Lo más difícil es conseguir que piensen un poco antes de contestar; suele haber competencia por contestar el primero.»

El profesor «artesano» presenta su metodología como «activa», con grandes dosis de improvisación en la que «el hacer» es el hilo conductor de la marcha del alumno, pequeño grupo de alumnos o grupo-clase. No por ello deja de articularse una participación con preguntas en explicaciones del profesor, c incluso, la adecuación del uso de la clase magistral.

«Uso mucho las preguntas para llevarios al lugar adecuado [...] $\mathrm{y}$ a veces cambio el fenómeno que analizamos si con ello consigo que quede más claro el camino que estamos siguiendo.»

«Normalmente me dirijo a todo el grupo, aunque los tengo organizados en grupos de cara al trabajo en el laboratorio y al material que tengo. Los grupos, los forman ellos a principio de curso y ya no cambian; suelen ser alrededor de seis equipos de cinco o seis alumnos."

Las fuentes de información que utiliza el alumno son diversificadas. Disponen de manuales y documentos aportados por el profesor y por los alumnos. El profesor utiliza el cuaderno del alumno como elemento para el trabajo en clase y para la evaluación. Y se apoya, a veces, en fotocopias de elaboración propia para complementar el trabajo en la pizarra y en el laboratorio.

«Al principio de cada tema les doy a los alumnos ideas del tema que vamos a trabajar. A veces se incluye sobre la marcha alguna cosa más, pero normalmente con eso sc pucde hacer todo el trabajo.»

La concepción de las actividades es de tipo muy abierto y sin secuencia alguna. Junto con las explicaciones del profesor se intercalan actividades y viceversa. En las experiencias de laboratorio, el profesor pretende, con los alumnos que están a su alrededor, seguir un modelo empírico-deductivo.

El trabajo práctico tiene un cierto toque empirista y se intercala continuamente en la explicación, para apoyarla e ilustrarla. Es frecuente que, ante una duda de los alumnos o una división de opiniones sobre cómo suceden las cosas, se improvise un montaje que muestre una situación de la cual inducir una conclusión más general. Este profesor no es exigente en necesidades de recursos, se adapta bien a los materiales que consiguen él y sus alumnos y por ello tiene gran variedad y flexibilidad en la elaboración y uso de los recursos de los que dispone. La utilización de cuestiones sobre el contex to y el entorno, planteadas como problemas de fácil solución, es una estrategia de uso común.

«Suelo preparar una demostración magistral cuando me interesa que vean algún fenómeno que muestre lo que cstamos estudiando o cuando no merece la pena emplear mucho tiempo en él. También si es algo que necesita un montaje complejo, o tengo poco material, o es peligroso.»

«Siempre que es posible, prefiero que utilicen matcriales de bajo coste o de desecho para los montajes experimentales. Mucho material específico de laboratorio es para el alumno una "caja negra" que tiene mucho más de magia que de ciencia.»

«Las prácticas de laboratorio que vienen con los equipos del Ministerio suelen ser complicadísimas y no aportan gran cosa, pero esos mismos equipos utilizados de otra manera son muy útiles. Es cuestión de adaptarlos a las necesidades de uno. 


\section{EI profesor «descubridor»}

El modelo de docente «descubridor» ha sido el emblema de la renovación durante los últimos años, con una imagen de estar en vanguardia, muy por delante de los demás modelos y haciendo gala de su planteamiento gobernado por la iniciativa de los alumnos.

Enraizado en una idea positivista de Ia ciencia, se caracteriza por postular un método científico empirista e inductivo y en tener como meta el descubrimiento investigativo. La enseñanza se entiende como descubridora de todo el conocimiento. Considera a los estudiantes como pequeños investigadores novatos que pueden obtener todas las leyes importantes a través del contacto y la observación directa de la realidad.

Con estos presupuestos, la planificación no sigue los contenidos de la materia disciplinar, sino que establece una serie de investigaciones de larga duración en las que el alumno reconstruirá, por descubrimiento libre, las principales bases de la ciencia.

«Normalmente se negocia con los alumnos los temas sobre los que se va a trabajar y, a partir de éstos, se producen ramificaciones que nos van permitiendo entrar en las distintas partes de la disciplina, aplicadas a lo que estamos estudiando".

«No suele dar tiempo de hacer sino un par de investigaciones por trimestre, pero creo que la profundidad y el interés con que se estudian compensan el no extenderse tanto».

La comunicación entre los alumnos es mucho más frecuente que con el profesor. Éste suele pasar por los grupos de trabajo de forma rotatoria. Así recibe información sobre la marcha del trabajo y facilita el material o la información solicitada por los alumnos. En la interacción se procura que el avance se haga sin ta intervención del docente.

«Una vez puesta en marcha una investigación, mi papel es el de facilitarles la tarea, conseguir el material necesario y orientarles en dónde buscar información, pero evito en lo posible que hagan algo porque yo sc lo haya sugerido."

«A veces se atascan y les ayudo a conseguir que se desatasquen, o llegan a un callejón sin salida o a comprobar que una de sus hipótesis era errónea y entonces mi papel es el de animador, el de haceries ver que de ese aparente fracaso también se sacan conclusiones y conocimicntos.»

Se sigue una metodología basada en el método de proyectos o de centros de interés, en la que el profesor es el animador y el ayudante; prepara al alumno, lo coloca en situación de rehacer los descubrimientos de las ciencias.

«l.o que se aprende es lo que se hace. Es inútil trabajar sobre asuntos que los alumnos no puedan tocar u observar directamente, ya que, si eso ocurre, será un asunto alejado de su entorno y sin interés para ellos."

«El método científico se aprende practicándolo. No podemos pretender que aprendan a resolver las situaciones reales si el trabajo de Iaboratorio consiste en seguir la receta que tienen preparada; son ellos quienes deben elaborar su investigación.»
«Lomás difícil es que lleguen a sus conclusiones. Frecucntemente debo intervenir para concretarles qué es lo que se puede deducir de lo que han hecho, bien mediante preguntas, bien mediante una explicación.»

Los documentos que utiliza este docente son variados. Dispone en el aula de información genérica y de libre acceso para los alumnos, y los recursos materiales se adaptan al trabajo de investigación en un aula-laboratorio preparada al efecto.

«Usamos todo lo que encontramos: como los temas los proponen cllos, suelen aportar su propio material y cómo usarlo.»

«Muchas veces panfletos o revistas tienen información muy preciada por ellos y que sirve perfectamente para nuestros fines."

La realización de pequeñas investigaciones de larga duración supone que la búsqueda de información y el trabajo práctico son las principales actividades. Los alumnos, individualmente o en grupos, solucionan por su cuenta problemas, cuestiones o experiencias, y recogen datos de los que inducir las leyes que los rigen.

El trabajo práctico no se asocia a un recinto cerrado, y se practica frecuentemente la docencia en la naturaleza (aulas de la naturaleza, aulas del mar, trabajos de campo, etc.) o en el entorno (arqueologia industrial, itinerarios tecnológicos, etc.).

«En el laboratorio hay mucho material que a ellos no les dice nada. Los aparatos construidos por ellos mismos tienen mucho más atractivo y les inicia en la tecrología con más eficacia. Y tienen muchísima imaginación construyendo cosas, más que uno mismo.s

«Para estudiar una hipótesis, intentamos probarla inmediatamente en el laboratorio. Eso refuerza mucho sus procedimientos, la práctica del método científico: si no sabes to que sucede, reprodúcelo y obsérvalo en el laboratorio."

\section{EI profesor «constructor»}

El modelo del docente «constructor», tal vez por ser el cronológicamente más reciente, es aún escaso; normalmente se encuentra vinculado a grupos de trabajo alrededor de algún proyecto o bien es un estudioso de los adelantos pedagógicos actuales.

El planteamiento básico del que parte es que el punto clave del aprendizaje es la mente del que aprende; por tanto, el diseño de todo proceso educativo tiene una base fuertemente condicionada por la psicología del aprendizaje y, dentro de ésta, por las teorías constructivistas del aprendizaje.

El profesor ayuda a la producción de conocimientos del alumno sin seguir un método científico inductivo, sino facilitando un cambio conceptual por avance graduat.

Con esta base, más que una programación, se sigue una planificación negociada «del todo» que suele ser interdisciplinar con tendencia a la integración. Este contrato 
no afecta únicamente al qué estudiar, sino que incluye aspectos como la distribución de las relaciones de poder dentro del aula, o mecanismos de evaluación que abarcan todos los componentes educativos, puesto que se considera que todos estos aspectos juegan un papel fundamental en los procesos del aprendizaje en las cabezas de sus alumnos.

«Detectar lo que piensan y qué conceptos erróneos tienen noes diffcil cuando coges práctica; to difíctl es planificar qué actividades les van a plantear un choque, la puesta en duda de lo que piensan y qué planteamientos y en qué momento les van a hacer cambiar de opinión, de teoría.»

El profesor coordina a los alumnos suministrándoles ideas, explicaciones y material necesario para las acciones que surgen. Tiene definido el camino que quiere seguir pero orienta más que gứa, por lo que el avance es lento.

«Sus líneas de razonamiento les llevarían frecuentemente a callejones sin salida y eso hace que muchas veces deba intervenir, llevándoles a detectar lo que está mal planteado o lo que conduce a ninguna parte. Muchas veces tengo la tentación de dejar que se estallen, pero pocas veces puedo permitírmelo: es muy caro en tiempo y en frustración."

«T tenen mucha tendencia a usar máquinas para todo: necesitamos un aparato que mida tal o una máquina que haga tal. Hay que empujarles a que desarrollen su trabajo conel material disponible, haciéndoles ver qué material es el idóneo para llevar a cabo su idea..

En este caso hay un papel de comunicación multidireccional entre docente y alumnos y entre estos últimos; eI docente es el coordinador del funcionamiento de la clase, dirige las situaciones y, al mismo tiempo, las modifica por la interacción con los alumnos, con la consiguiente pérdida de protagonismo en el desarrollo de Ios acontecimientos.

La clase se organiza de común acuerdo con los alumnos, en grupos variables y pequeños, con previsión de dinámicas de cambio de sus elementos.

«Mucho tiempo se consume en sus propias discusiones, mi papel es más intenso cuando se bloquean para intentar orientarles. Suelo ir pasando por los grupos y ellos aprovechan para ver qué me pueden sacar sobre la marcha. Pensar es demasiado fatigoso, pero les encanta cuando desarrolian e imponen una teoría con argumentos que no se pueden rebatir. La competitividad entre grupos sustituye a la competitividad entre individuos.»

Los locales de aula, aula-laboratorio o cualquier otro, con el material consiguiente, se usan con gran flexibilidad y se eligen libremente.

«Trabajamos en el laboratorio, usando las mesas como pupitres y superficie de trabajo al mismo tiempo. Ellos saben dónde está cała material, así que se sirven ellos mismos lo que necesitan.»

De este modo, la biblioteca del aula juega un papel muy importante para que el alumno tenga acceso a libros y documentos variados. El cuaderno de aula del alumno es un elemento clave en las estrategias de enseñanzaaprendizaje.
«Su cuaderno es su almacén de información y deben saber organizarlo y mantenerlo. Ahí tienen acumulado lo que han hecho, lo que han aprendido y lo que saben, sus errores y como corregirlos. Refleja todo su trabajo y es el mejor espejo en que se pueden mirar y en donde yo les miro.»

La metodología que utiliza este modelo de profesor es investigativa, basada en la "resolución de problemas» abiertos, entendidos como dificultades sin solución inmediata. Las clases tienen una gran actividad por practicar un cierto descubrimiento guiado para conseguir una elaboración del conocimiento. En ocasiones, se intenta resolver problemas que no tienen solución.

«Hay muchos problemas que dan abundante juego para esiudiar ciencia formal: cómo colgar una hamaca, cómo separar el aderezo de una ensalada, cómo funciona una aspirina efervescente, etc., sun problemas que exigen mucha física, mucha química y mucho procedimiento de investigación."

El profesor encarga diseños y sugiere actividades y experiencias relacionadas con el trabajo, para poderlas llevar a cabo en el aula o en el entorno o, incluso, en el laboratorio. Así, a veces, algunos alumnos diseñan lo que hacen, otros eligen diseños orientativos y a los menos se les dirige a la hora de elegir su ritmo.

«Muchas veces se les ocurren métodos que a mi no se me habrían pasado por la cabeza y que funcionan: unos alumnos me separaron agua y aceite congelándolos y usando un cuchillo para recoger el aceite como si fuera mantequilla."

Este modelo puede hacerse corresponder con lo que se ha dado en Ilamar «modelo constructivista». Sin embargo, no hay unanimidad entre diversos autores a la hora de definirlo: "Y ahora que todos somos constructivistas, ¿qué?; pero, ¿de qué constructivismo se habla?... porque parece que hay muchos constructivismos (Gómez-Graneil, 1993).

En suma, este modelo pretende que los alumnos sean protagonistas de su aprendizaje; sin embargo, para que tal situación se dé, es necesario una adecuada y difícil tarea de dirección por parte del profesor (Porlán, 1993).

\section{MODELOS Y DIVERSIDAD}

Una revisión de la tabla I permite observar los distintos planteamientos que tienen los diferentes modelos didácticos sobre cada aspecto del "cómo enseñar».

Creemos que, al igual que en un ecosistema, la predominancia de una especie supone una menor abundancia de especies en todo el sistema; por tanto la actual preponderancia del modelo transmisivo o una posible futura predominancia de otro modelo, suponen una diversidad escasa y, con ello, una pobreza dei sistema educativo.

Pensamos que no se puede desechar sin matices ningún modelo y que, de hecho, todo docente utiliza varios en función de las circunstancias. Sería deseable que todo docente estuviera en disposición de utilizar lo que cada 
modelo aporta de positivo y que fuera consciente de las limitaciones y de los efectos no deseados que cada modelo puede provocar.

Defendemos esta postura ecléctica por ser la más factíble y de mayor potencial de cambio, la más respetuosa con la personalidad y la historia de cada individuo y la que aporta más riqueza al ecosistema educativo.

\section{AIGUNAS SUGERENCIAS}

Tal como se indicó anteriormente, buscamos con este estudio el desarrollo de una herramienta de análisis del pensamiento y la práctica docente que nos permita fundamentar el desarrollo profesional docente. Incidimos en «cómo enseñar» $y$, en parecidos términos, se puede hablar de "cómo evaluar», "cómo ampliar la propia formación», «cómo se ve a los alumnos» y demás facetas de una cuestión tan compleja como es la docencia. Una vez dispusiéramos de esta herramienta, ¿qué haríamos con ella?.

\section{REFERENCIAS BIBLIOGRÁFICAS}

BRINCONES, I. et al. (1986). Identificación del comportamiento y características deseables del profesorado de ciencias experimentales en bachillerato. Enseñanza de las Ciencias, 4(3) pp. 209-222.

CAÑAL, P. y PORLÁN, R. (I987). Bascs para un programa de investigación en tomo a un modelo didáctico de tipo sistemático e investigativo. Actas del II Congreso Intemacional sobre Investigacion en la Didáctica de las Ciencias y de las Matemáticas. Valencia.

CAÑAI, P. y PORLÁN, R. (1987). Investigando la realidad próxima: un modelo didáctico alternativo. Enseñanza de las Ciencias, 5(2), pp. 89-96.

GARCÍA, J.E. (1986). Bases para la introducción del modelo de profesor-investigador en los centros de profesores. Actas IV Jornadas de Estudio sobre Investigación en la Escuela. Sevilla.

GARCÍA, J.E. (1988). Fundamentos para la construcción de un modelo sistémico de aula, en Porlán, R. y Coll, C., Constructivismo y enseñanza de las ciencias. Sevilla: Díada liditores.

GIL. PÉREZ,D. (1983). Tres paradigmas básicos en la enseñanza de las ciencias. Enseñanza de las Ciencias, 1(1), pp. 26-33.

GIL PEREZ, D. (1993). Contribución de la historia y de la filosofía de la ciencia al desarrollo de un modelo de enseñanzaaprendizaje como investigación. Enseñanza de las Ciencias, $11(2)$, pp. 197-212.

GIMENO SACRISTÁN, I. (1981). Teoría de la enseñanza y desarrollo curricular, cap. 3, pp. 104-152. Madrid: Anaya.
Para ei docente, en general, disponer de modelos didácticos puede tener varios usos. Puede ser útil para explícitar un posicionamiento personal que ponga de manifiesto las virtudes y los defectos de la propia práctica y que, al mismo tiempo, ilumine los presupuestos teóricos que la sustentan. Otro uso es el de permitir la prospección de otras posibilidades de teoría o práctica profesional de cara a la innovación y a la evolución del quehacer propio. Muchas veces, la convicción de la bondad de las posiciones personales está basada en el desconocimiento de otras alternativas.

Para el asesor de profesores, la propuesta de análisis de los diferentes modelos a un grupo de docentes puede ser la vía de explicitación de las posiciones y convicciones de los miembros del grupo. En el análisis de situaciones de este tipo, hemos observado que cada cual tiende a identificarse con los presupuestos de uno o dos modelos y a criticar los demás. Probablemente con el trabajo de crítica y comparación de los modelos didácticos se obtenga más información de la propia imagen que de «lo que verdaderamente pensamos y hacemos» pero, aun así, éste es un buen punto de arranque para reflexionar sobre cómo enseñar.

GÓMEZ-GRANELL, C. y COLL SALVADOR, C. (1993). ¿De qué hablamos cuando hablamos de constructivismo? Cuadernos de Pedagogía, 22I, pp. 8-10.

HOST, V. (1988). «Caracterización de modelos didácticos en la enseñanza de las ciencias». Ponencia del curso de Formación de Formadores. Sevilla.

MARTÍNEZ SANTOS, \$. (1989). Estructura curricular y modelos para la innovación. Ed. Nieva.

PORLÁN ARIZA, R. (1986). La epistemología del profesor de ciencias: una investigación en curso. Actas IV Jomadas de Estudio sobre Investigación en la Escuela. Sevilla.

PORLÁN ARIZA, R. y FLOR PÉREZ, J.I. (1983). «El modelo sistémico investigativo». Conferencia en el CEP de Santander, marzo 1993.

PO70, I. (1987). Y sin embargo se puede enseñar ciencia. Infancia y Aprendizaje, 38, pp. 109-113.

ROSADO BARBERO, L. (1979). Didáctica de la fisica. Zaragoza: Luis Vives.

ROZADA MARTÍNEZ, J.M. (1985). Análisis del modelo didáctico de la reforma. Cuaderno $n^{\circ} I:$ : $l$ modelo didáctico: Reformas en EGB y Medias. Gijón: CEP.

RO7.ADA MARTÍNEZ, J.M. (1985). Enseñar a investigar. La necesidad de un modelo didáctico. Escuela Asturiana, 13 , pp. 5-6.

TOULMIN, S. (1977). La comprensión humana, Vol. I: El uso colectivo y la evolución de los conceptos. Madrid: Alianza Editorial. 
Para saber más, conviene conocer:

AUSUBEI, D.P. (1978). Psicología educativa: un punto de vista cognitivo. México: Trillas.

CASCANTE, C. y ROZADA, J.M. (1987). Proyectos curriculares y formación en el trabajo. Cuadernos de Pedagogia, 168, pp. 28-32.

ELLIOT, J. (1988), «Diseño de una actividad de asesoramiento desde la perspectiva de la investigación acción». Ponencia del curso de Formación de Formadores. Sevilla.

ESCUDERO MUÑOZ, J.M. (1981). Los modelos didácticos. Barcelona: Oixos-Tau.

FAVIERFS, A., MANRIQUE, M.J. y VARELA, P. (1985). Evvaluación comparativa de dos métodos de trabajoen química de $2^{\circ}$ de BUP. Enseñanza de las Ciencias, núm. extra, p. 42.

GIMENO SACRISTÁN, J. (1982). La pedagogía por objetivos, una obsesión por la eficiencia. Madrid: Morata.

GIMENO SACRISTÁN, J. (1988). Teoría de la enseñanza y desarrollo del currículo. Madrid: Anaya.
JOICE, B. y WEIL, M. (1985). Modelos de enseñanza. Madrid Anaya

LOPEZ HERRERÍAS, J.A. (1978). Tipos de enseñanzaaprendizaje y su valor didáctico: dogmática, escéptica y crítica. Revista de Bachillerato, 6, pp. 15-25.

PALACIOS, C., MUÑOZ, P. y GÓMEZ, J.C. (1985). Dos metodologías activas comparadas en el estudio de conceptos químicos en $8^{\circ}$ de EGB. Enseñanza de las Ciencias, núm. extra, p. 43.

POPE, M. (1988). «Estrategias de Formación deł Profesorado». Ponencia del curso de Formación de Formadores. Sevilla.

POPE, M. y SCOTT, E. (1988). «La epistemología de los profesores y la prácticas. Ponencia del curso de Formación de Formadores. Sevilla.

SOIÍS VILLA, R. (1984). Ideas intuitivas y aprendizaje de lat ciencias. Enseñanza de las Ciencias, 2(2), pp. 83-89.

[Artículo recibido en marzo de 1995 y aceptado en mayo de 1996.] 